\title{
Tangence
}

\section{Les frontières du hasard : de la fiction à l'Histoire}

\section{Jean-François Chassay}

Numéro 43, mars 1994

Babel et après : Paul Auster

URI : https://id.erudit.org/iderudit/025801ar

DOI : https://doi.org/10.7202/025801ar

Aller au sommaire du numéro

Éditeur(s)

Tangence

ISSN

0226-9554 (imprimé)

1710-0305 (numérique)

Découvrir la revue

Citer cet article

Chassay, J.-F. (1994). Les frontières du hasard : de la fiction à l'Histoire.

Tangence, (43), 51-67. https://doi.org/10.7202/025801ar d'utilisation que vous pouvez consulter en ligne.

https://apropos.erudit.org/fr/usagers/politique-dutilisation/ 


\section{Les frontières du hasard: de la fiction à l'Histoire}

Jean-François Chassay

Il y a un plaisir piquant à se tourner vers le passé, à se demander "Que serait-il arrivé si..." et à substituer un effet de hasard à un autre. On observe ainsi comment, d'un moment gris, stérile et ennuyeux de notre vie s'épanouit un magnifique et riant événement qui, en fait, n'a pu éclore. Elle est mystérieuse, cette structure en carrefour de la vie: on aperçoit, à chaque instant du passé, un choix entre deux directions, un "c'est ainsi" et un "autrement ", d'où les innombrables et vertigineux zigzags, bifurcations et trifurcations qui s'esquissent sur le fond sombre du passé.

Vladimir Nabokov,

Le guetteur

Comment s'étaient-ils rencontrés? Par hasard, comme tout le monde.

Denis Diderot, Jacques le fataliste

Paul Auster reçoit parfois un accueil mitigé aux États-Unis, pour des raisons que d'aucuns trouveront étonnantes. On lui reproche notamment d'accorder trop de place aux hasards dans son œuvre romanesque, ceux-ci n'apparaissant pas, à certains, suffisamment "réalistes". Dans ses livres, les connexions provoquées par le hasard romanesque attirent l'attention sur de "l'étrangeté", du sens en surcroît, superfétatoire sans doute pour certains critiques qui ressentent dans cette organisation narrative trop d'effets littéraires (comme si la littérature ne devait pas être littéraire...). 
L'idée même de "réalisme" (dont on ne soulignera jamais assez la complexité) conduit souvent à d'aberrantes réductions. Dans un article publié récemment, Régine Robin démontrait, exemples à l'appui, que ce rapport au hors-texte rendait difficile, pour sociologues et historiens, l'interprétation du texte littéraire. Deux sociologues s'étonnent:

L'un des suicides littéraires les plus célèbres est celui d'Emma Bovary; c'est aussi l'un des plus improbables. Femme, jeune, mariée, mère d'un enfant, rurale et catholique, elle cumulait les traits dont Durkheim a montré qu'ils constituaient les facteurs les plus efficaces de la préservation du suicide. [...] D'après Durkheim, Emma Bovary avait moins de 74 chances sur 100000 de se suicider, alors que Rodolphe en avait lui plus de 985. Flaubert s'étend sur le premier et ne souffle mot du second, ô combien plus commun. Or, l'ambition sociologique du romancier ne fait aucun doute [...].

Comment diantre un homme intéressé par des données sociologiques peut-il faire fi des statistiques? Un portrait "réel" de la province française aurait mérité, à tout le moins, un deuxième suicide en prime. Un historien, de son côté, insiste: Stendhal dans Le Rouge et le Noir et Flaubert dans L'éducation sentimentale font des erreurs de date, passent à côté d'événements politiques ou économiques majeurs ${ }^{1}$. Pourquoi ne pas suivre la chronologie? Pourquoi ne pas tout expliquer? Salman Rushdie, quant à lui, rappelle dans un article avoir reçu de nombreuses lettres de lecteurs fiers de leur perspicacité et essuyé les commentaires de nombreux Hindous courroucés, devant les "erreurs historiques" que multiplient Les enfants de minuit ${ }^{2}$. De la même façon que certains traquent les signes de l'autobiographie au moindre détour des phrases, d'autres ratissent l'espace historique et géographique pour s'assurer que la fiction se conforme au réel, que le "vécu" des personnages romanesques correspond scrupuleusement au "vécu" des individus dans la "vraie vie". À bas le hasard, ce qui grinche et dérape, vive la tautologie.

Évidemment, on pourra arguer que si la littérature se contentait de reproduire les faits historiques, de rendre compte, à travers

1 Voir Régine Robin, "Pour une socio-poétique de l'imaginaire social", Discours social/Social Discourse, vol. V, $\mathrm{n}^{\mathrm{os}}$ 1-2, hiver-printemps 1993, p. 25-26.

2 Salman Rushdie, "Errata ou la narration trompeuse dans Les enfants de minuit", dans Patries imaginaires, Paris, Bourgois, 1993, p. 33-37. 
des archétypes "raisonnables", de statistiques et sondages sous prétexte d'objectivité, elle répéterait, balbutierait les sciences humaines et deviendrait obsolète. La coalescence entre le texte littéraire et le discours social dans lequel il baigne n'implique en aucune façon la parfaite adéquation entre la diégèse romanesque et la société réelle. Dire le contraire impliquerait la nécessité de se conformer à une lecture sociologisante. Or, personne ne demande à l'écrivain d'être sociologue. La littérature "jouit du droit, écrit Erich Köhler ${ }^{3}$ dans Le basard en littérature, de faire abstraction, totalement ou partiellement, du possible réalisé empiriquement et de le remplacer par le possible qui ne s'est pas du tout ou insuffisamment réalisé en tant que nécessité - parvenue à l'existence dans la seule fiction, il est vrai, mais contenue dans le réel ".

La valorisation du hasard, de la "non-concordance", de la subjectivité, le refus des faits pour eux-mêmes, conduisent justement à indiquer certaines modalités de la fiction et une forme de savoir qui lui appartiendrait en propre. Si le texte littéraire a une valeur cognitive au-delà de ce qu'il raconte, c'est justement parce qu'il ne répond pas aux balises de l'objectivité scientifique. Ou plutôt : parce qu'il met en doute une forme de raisonnement qui reposerait entièrement sur l'objectivité scientifique. Non pas parce que le texte littéraire défend les grandes majuscules (Création, Génie, Inspiration, propulsant le lecteur dans une vaporeuse immanence), mais parce qu'il questionne la nature même de la réalité, les évidences souvent trompeuses. C'est cette "étrangeté " par rapport au réel qui donne son poids épistémologique aux textes de Paul Auster.

Si la place de l'aléatoire ne se dément pas dans son ouvre fictionnelle depuis ses premières publications, elle prend une coloration particulière dans Moon Palace (et dans une moindre mesure dans Leviathan que je n'aurai pas le temps d'examiner ici), dans la mesure où, à travers la diégèse romanesque, se manifeste un discours de et sur l'histoire américaine. Ainsi l'importance de la filiation, flagrante dès L'invention de la solitude, ne concerne plus seulement le père génétique mais aussi les "Pères de la nation. Et puisqu'il est question de filiation, rappelons que la présence du hasard chez Auster concerne de près l'identité. Cela ne saurait surprendre:

Erich Köhler, Le basard en littérature, Paris, Klincksieck, 1986, p. 104. 
54

L'édifice de la science comme l'histoire humaine incorporent une large part d'arbitraire, et l'on se prend à rêver à ce qui aurait pu être, mais qui n'a finalement pas été. Nous sommes les survivants d'un impitoyable processus de sélection, qui choisit dans l'infinie variété des futurs possibles celui qui finalement se réalisera. [...] Rien d'étonnant dès lors à ce que celui qui se laisse aller à ces pensées subisse une crise d'identité: qu'est-ce que ce monde si contingent, qu'est-ce que ce moi devant tant d'autres existences virtuelles? ${ }^{4}$

Au bout du compte, la notion d'un savoir propre à la littérature se voit interrogée à travers ces hasards. Car ce que tisse Paul Auster dans son texte, c'est une histoire en abrégé de l'Occident, faite de rencontres, de télescopages, de retournements, qui culmine dans la conscience et même dans le nom du narrateur. Marco Stanley Fogg, figure brouillée ${ }^{5}$ de l'aventurier parti de l'Europe pour découvrir le monde (en Asie dans le cas de Marco Polo, en Afrique dans celui de John Rowlands Stanley), aboutit au patronyme de celui qui fit le tour de la planète: Philéas Fogg ${ }^{6}$. Il devient fil conducteur d'une conscience en plein brouillard, en quête de sens dans un monde échappant à tout contrôle, dans une société américaine où l'amnésie est fréquente. Fogg luimême parle de "la sensation de l'infinie fragilité de [s]on nom"?. Cet individu toujours au bord de la catastrophe ressemble à un pays, à une société, elle-même fort sensible à la déroute (politique, sociale), et peut-être moins sûre d'elle-même qu'on le dit souvent.

\section{$\stackrel{* *}{* * *}$}

L'épigraphe de Moon Palace mérite une attention particulière: "Rien ne saurait étonner un Américain». Cette citation tirée

4 Ivar Ekeland, Au basard. La chance, la science et le monde, Paris, Seuil, 1991, p. 70 .

5 Fog (avec un seul g) veut dire brouillard et "to be in a fogg" se traduit par "ne plus savoir où on en est ", ce qui est bien le cas du narrateur dans ce roman.

6 Évidemment, le roman est écrit en anglais et "fil" ne se dit pas de la même façon dans la langue de Hawthome. Mais Paul Auster connaît bien le français et il est question ici du nom d'un personnage tiré d'un roman de Jules Verne, ce qui me paraît rendre défendable cette petite hypothèse.

7 Paul Auster, Moon Palace, Arles, Actes Sud, 1990, p. 18. Les citations du livre renverront toutes à cette édition française. 
du roman De la terre à la lune de Jules Verne indique un premier pont (littéraire dans ce cas-ci) jeté entre les États-Unis et l'Europe ("la rencontre de Spike Jones et de Schopenhauer", comme l'écrit Fogg à propos de son oncle Victor). Ce ne sera pas le seul, le roman en regorge: qu'on songe à Effing, personnage éminemment beckettien $^{8}$, ou encore à Pavel Shum, ce "transfuge " qui rappelle la figure de Nabokov. En effet, ce Russe blanc, érudit, parlant couramment six ou sept langues, fuyant la France juste avant l'arrivée des Nazis, devint, selon les propos d'Effing, "un maitre de la phrase poétique, un inventeur hors pair d'images appropriées et frappantes, un styliste dont les mots pouvaient comme par miracle révéler la réalité palpable des objets. "Et penser, ajoutait-il, que l'anglais n'était pas sa langue matemelle" (p. 150). Humbert Humbert, dans Lolita, comme Fogg, parcourt le territoire américain.

Outre ce premier lien littéraire esquissé entre deux continents, l'épigraphe semble faire écho aux propos de Fogg dans le roman: "la causalité n'était plus le démiurge caché qui régit l'univers [...] Héraclite était ressucité de dessus son fumier, et ce qu'il avait à nous montrer était la plus simple des vérités: la réalité était un yo-yo, le changement la seule constante" (p. 84) ${ }^{9}$. En même temps elle ne manque pas d'ironie, puisqu'elle indique a contrario l'état dans lequel se retrouve le narrateur, continuellement frappé d'étonnement. "La fonction de l'étonnement, écrit Aldo G. Gargani, est celle d'une mise en mouvement: lorsqu'on l'associe au vécu, au sens où celui-ci représenterait une entitée privée et inaccessible [...], il paraît être exactement le contraire de ce qu'il est. L'étonnement est l'état dans lequel un homme reconnaît qu'il n'est pas ce qu'il est ou croyait être. ${ }^{10}$ Or, l'errance volontaire de Fogg le conduit à disparaitre à ses propres yeux, c'està-dire, précisément, à ne plus être ce qu'il croyait être: "Je constatais le progrès de mon propre démembrement. Morceau par morceau, je me regardais disparaitren (p. 39); "À certaines périodes, je perdais carrément ma propre trace" (p. 46), au point que

8 On pourrait dire la même chose de Pozzi et Nash dans La musique du basard, doubles de Pozzo et Lucky.

9 Cela rappelle également l'épigraphe de L'invention de la solitude, tirée de l'œuvre d'Héraclite: "Qui cherche la vérité doit être prêt à l'inattendu, car elle est difficile à trouver et, quand on la rencontre, déconcertante." 
les lieux n'ont plus de sens: "C'était New York, mais cela n'avait rien à voir avec le New York que j'avais toujours connu. Cet endroit était dépourvu d'associations, il aurait pu se trouver n'importe où " (p. 76). Tout cela provoque paradoxalement un mouvement qui met le sujet en crise et entraîne une série d'étonnantes cö̈ncidences, modifiant radicalement le cours de son existence. Pour Gargani, "le hasard est une chose qui ressemble moins à ce qui se produit lorsqu'on trébuche sur une pierre qu'à ce qui caractérise, au contraire, la saisie d'une nouvelle possibilité au cœur d'une codification du monde et des situations ordinaires de la vie "11. En remettant en question son existence (et peut-être plus précisément sa raison), Fogg ouvre la voie aux possibles, à un nouveau monde de correspondance ("les correspondances infinies ", p. 27) où, pour reprendre une idée-force du mythe de l'Amérique mais dans un autre contexte, tout devient possible: "Dès lors qu'on a jeté sa vie à tous les vents, on découvre des choses qu'on n'avait jamais soupçonnées, des choses qu'on ne peut apprendre en nulle autre circonstance. (p. 79).

Il n'y a pas, il ne peut y avoir de séries causales indépendantes dans l'univers. Cependant, en provoquant une rupture radicale dans sa propre vie, Fogg devient en quelque sorte un clinamen, cette forme atténuée de liberté qu'Épicure accordait aux atomes, consistant dans leur capacité de modifier, même très légèrement, leur trajectoire. Le narrateur se révèle l'erreur dans le système qui déclenche le jeu des transformations, des coïncidences, qu'on appelle vulgairement des hasards. Chercher à isoler les causes d'un événement frappant est une démarche stérile car "une cause très petite, qui nous échappe, détermine un effet considérable, que nous ne pouvons pas ne pas voir, et nous disons alors que cet effet est dû au hasard " 12 .

Lieu du dédoublement et du redoublement, Moon Palace, comme son titre le laisse entendre, se trouve placé sous le signe de la lune. Renvoyant à un univers symbolique, la lune, très concrètement, permet également de rendre compte des derniers développements de la recherche technoscientifique. L'incipit du roman

11 Ibid., p. 37.

12 Henri Poincaré, cité par Evar Ekeland, Au basard, op. cit., p. 134. Ce qu'Effing traduit à sa façon: "Cette nuit-là a eu des conséquences [...]. C'est ainsi que ça se passe, mon garçon. Il y a toujours des conséquences, qu'on le veuille ou non* (p. 187). 
se réfère à la mission Apollo 11: "C'était l'été où l'homme a pour la première fois posé le pied sur la lune" (p. 11). Ouvrir le roman avec cette phrase indique d'emblée la place accordée à l'American Dream (et donc à l'histoire américaine), puisqu'elle renvoie au mythe de la frontière analysé par Frederick Turner. Après avoir traversé cette ultime frontière, celle de l'espace, que reste-t-il à faire? À ce propos, Salman Rushdie avance l'hypothèse suivante:

l'idée qu'un pays né avec le besoin de repousser ses frontières vers l'ouest a dû continuellement trouver de nouvelles frontières depuis qu'il a atteint le Pacifique est depuis longtemps utile pour regarder l'histoire américaine. La conquête de l'espace n'est qu'un des sujets éclairés par cette thèse. Dans les deux dernières décennies, beaucoup d'Américains se sont tournés à l'intérieur d'eux-mêmes à la recherche de cette nouvelle frontière. ${ }^{13}$

C'est à cette autre "frontière ultime", celle de sa conscience, que Fogg se voit confronté. Moon Palace vise à abolir l'espace entre ces deux frontières en faisant retraverser à son narrateur toutes les autres. Accomplir de nouveau le parcours de la nation, de la côte Est jusqu'au Pacifique, devient une tentative de déchiffrement de l'espace américain, une quête de savoir sur le monde permettant d'échapper au chaos en passant par celui-ci. "Il me semblait, dit Fogg, que si je m'abandonnais au chaos de l'univers, l'univers me révèlerait peut-être en dernier ressort une harmonie secrète, une forme, un plan, qui m'aideraient à pénétrer en moimême. La condition était d'accepter les choses telles qu'elles se présentaient, de se laisser flotter dans le courant de l'univers" (p. 104-105). Cette volonté de déchiffrement de l'univers (qui n'est pas sans rappeler les Romantiques allemands, Novalis en tête) forme le noud du roman.

En ce sens, la présence polysémique de la lune joue un rôle fondamental. Les références à la lune permettent de se confronter à des paysages, des objets, à tout un univers matériel et singulier au cours du roman. Mais elles renvoient également à un monde culturel, symbolique, marqué par la mort et la résurrection.

En 413 avant notre ère, le corps expéditionnaire athénien, écrasé à Syracuse, attribue sa défaite à un coup du sort: une

13 Salman Rushdie, "In God We Trust", dans Patries imaginaires, op. cit., p. 415. 
éclipse de lune a impressionné les soldats athéniens la nuit où ils devaient s'enfuir. Férus d'oracles et de divination, leurs chefs y voient le signe qu'ils doivent demeurer sur place. Ils seront massacrés. Cette marque d'un message divin pour les Athéniens nous apparaît à nous, qui connaissons la mécanique céleste et les lois de Kepler, comme un événement naturel où le hasard n'a pas sa place. "Nous touchons ainsi du doigt le fait que le hasard est toujours une réponse à une question qu'un homme se pose. " ${ }^{14} \mathrm{Ce}$ que nous appelons hasard apparait comme une clé, un signe permettant de répondre à une angoisse souvent diffuse.

Entre l'oracle et la réalité scientifique, les Athéniens ne pouvaient pas choisir car leurs connaissances des mouvements célestes étaient limitées. Il reste que la lune a toujours appelé les contradictions :

Un peu partout, la Lune connote le mystère, le romanesque, le bizarre: le capricieux est déclaré "lunatique n, le rêveur "dans la lune "(moonstruck) [...] Plus profond encore est le lien primordial entre la lune et la notion de mesure. Le mot anglais moon et ceux de la même famille dans les autres langues viennent de la racine $m e$, qui signifie mesure (dans metron en grec, par exemple, ou dans meter et measure en anglais), et nous rappelle que l'astre nocturne a été le premier garde-temps universel. ${ }^{15}$

Oracle et science, poésie et mesure mathématique, bizarrerie et régularité: l'astre qui domine la terre est le lieu de toutes les contradictions, du rationnel à l'irrationnel, de la sagesse à la folie. Sous l'influence de la lune, Moon Palace, roman déconcertant, apparaît marqué par le contraste entre un univers extrêmement méthodique, chiffré, cartographié, et l'espèce de torpeur dans laquelle les personnages - et, au premier chef, le narrateur semblent se mouvoir, comme si la réalité n'existait que par ce qu'elle a d'incontrôlable, d'inévitablement aléatoire. De la date de sa convocation militaire pour l'examen médical à la découverte de son père, de la mort accidentelle de sa mère au fait que Kitty et Zimmer réussissent à retrouver sa trace, de la découverte dans le désert d'une caverne par Effing qui lui sauve la vie à la gros-

14 Ivar Ekeland, Au basard, op. cit., p. 145. L'anecdote qui précède est également tirée de ce livre. Voir p. 141-145.

15 Daniel Boorstin, Les découvreurs, Paris, Robert Laffont, coll. "Bouquins ", 1986, p. 9. 
sesse de Kitty Wu, les événements vécus (ou connus) par Fogg font sens tout en n'ayant aucun sens. Rien n'est lié et finalement tout se lie. Entre l'Histoire et son histoire, entre le monde et lui, la brèche s'amplifie et se comble au gré des événements.

Il ne faut pas s'étonner dans ce contexte des nombreuses allusions au baseball, surtout en cette année 1969. Le baseball est un sport où le temps ne se découpe pas, s'écoule sans contraintes, jusqu'à la victoire finale d'une équipe sur une autre. Il repose sur une temporalité "naturelle" en quelque sorte, lunaire pourraiton dire. Sport reposant à l'extrême sur les infimes détails et la précision ${ }^{16}$, il dépend du hasard plus que tout autre (un ballon ou une chandelle perdus dans le soleil, un signal mal compris par le frappeur...). Mais 1969, c'est d'abord l'année de ceux qu'on a nommé les Miracle Mets (encore un miracle, encore un oracle). “À vrai dire, écrit Fogg, je me sentais plutôt gratifié par la bonne étoile des Mets. [...] Les voir soudain, contre toute vraisemblance, surgir des profondeurs, cela semblait prouver que tout en ce monde était possible" (p. 83). Cette situation apparaît inadmissible, inimaginable, non seulement à cause de la rapide remontée des Mets en septembre qui leur permettait de devancer les Cubs de Chicago, mais surtout parce que cette équipe, cinq ou six ans plus tôt seulement, impressionnait d'abord par sa médiocrité. À l'image de ces conscrits recalés parmi lesquels se trouve Fogg, les Mets quelques années auparavant semblaient composer avec "les demeurés, les grotesques, des jeunes gens qui n'avaient nulle part leur place" ${ }^{17}$ (p. 106). En 1969, alors que la guerre du Viet-Nam tourne au vinaigre après My-Laï, au moment du procès des huit de Chicago et des Black Panthers, voilà que les Mets détrônent leurs puissants voisins du Bronx au passé glorieux et mythique (et au nom révélateur), les Yankees. On ne pourrait manifester de manière symbolique plus forte la chute que subissait l'image des États-Unis sur terre alors que la nation levait les yeux vers la lune. Et il est significatif également que la cuisante déroute survienne à une équipe de Chicago, en l'occurrence les Cubs: "Pour comprendre le gigantesque "en avant", la trajectoire qui longtemps

16 Voir par exemple à ce propos le livre de George Will, Men at Work, New York, Harper Perennial, 1991, IX/353 p., détestable idéologue de droite mais fin connaisseur de baseball.

17 Formule qu'on pourrait aussi utiliser pour parler de la célèbre équipe que présente Philip Roth dans The Great American Novel. 
60

résuma l'Amérique, c'est toujours, disait Henry Adams, de Chicago qu'il faut partir." ${ }^{18}$ Comme c'est souvent le cas chez les écrivains américains, chez Paul Auster le baseball n'est pas qu'un jeu, un sport, mais une métaphore ou une métonymie, selon les cas, traduisant des conflits et des tensions politiques.

La structure narrative rend compte également de cet univers contrasté où raison et déraison s'imbriquent parfaitement. Dès le premier paragraphe, le narrateur annonce, en accéléré, le programme du livre et toutes les révélations qui vont suivre. Il s'agit davantage d'un télescopage d'informations alors sans signification pour le lecteur plutôt que d'un véritable "résumé" qui mettrait les choses en place. Néanmoins, cette stratégie narrative se répétera régulièrement au fil des pages. Les nombreuses prolepses manifestent une volonté de rationalisation, dans l'après-coup, d'événements surgissant comme de véritables miracles (petits ou grands) dus au hasard. Par exemple, alors qu'il fait ses adieux à Zimmer dont le lecteur n'entendra plus parler dans le roman, Fogg s'empresse d'ajouter qu'il le rencontrera treize ans plus tard par hasard (évidemment...) à New York. Cette scène décontextualisée, hors propos, propulse le lecteur dans une espèce de hors-texte (fictif) qui indique que les fils de la "réalitée" (fictionnelle, bien sûr) ne sont pas complètement rompus. Les coïncidences étonnantes sont ainsi réinterprétées et présentées par un homme ayant traversé une quête initiatique et remettant en ordre les pièces du puzzle comme si elles trouvaient leur place dans un univers entièrement déterministe. Et dans une certaine mesure, l'histoire de Fogg est effectivement entièrement déterminée, puisqu'elle se superpose en grande partie à l'histoire américaine ${ }^{19}$.

Le premier Fogg, un Fogelman en réalité, arrive en Amérique par la voie obligé d'Ellis Island. La transformation de son nom, par un fonctionnaire analphabète ou paresseux, est déjà une indication de la rupture avec l'ancien monde. Mais c'est aussi un premier

18 Pierre-Yves Pétillon, Histoire de la littêrature américaine. Notre demi-siècle 1939-1989, Paris, Fayard, 1992, p. 694.

19 Histoire éclatée bien sûr, qui ressemble à la lecture de Fogg des livres de son oncle: "C'était presque comme parcourir l'itinéraire d'un explorateur du temps jadis, retracer les pas qui l'avaient porté dans un territoire vierge [...] Le voyage consistait donc en excursions discrètes, discontinues. De Boston à Lenox, par exemple. De Minneapolis à Sioux Falls. De Kenosha à Salt Lake City. Peu m'importait d'être obligé de sauter d'un bout à l'autre de la carte. À la fin, tous les vides seraient remplis, toutes les distances couvertes" (p. 36-37). 
signe du hasard, ou plutôt de l'arbitraire, qui s'inscrit jusque dans son patronyme. Ce ne sera pas la seule apparition de l'arbitraire dans sa vie: événement central et déterminant, sa mère meurt accidentellement à Boston, lieu d'un acte fondateur, d'une rupture déterminante dans l'histoire américaine, à savoir le Boston Tea Party. Il retrouve son oncle Victor à Chicago, ville dont le développement tardif mais rapide, au milieu du $\mathrm{XIX}^{\mathrm{e}}$ siècle, en fera un centre essentiel du Midwest. C'est d'abord par le biais de celui-ci qu'il fait la conquête de l'Ouest (puisque son oncle effectue des tournées au sein d'un groupe, comme clarinettiste: "Nous mettons le cap à l'ouest et nous lançons dans les régions sauvages", p. 25), puis grâce au récit (peut-être véridique) d'Effing, ensuite à celui (fictif) de Barber, avant de traverser lui-même ce paysage lunaire (les vastes plaines, légèrement déprimées, plus ou moins ondulées et bordées de montagnes qu'on retrouve en Utah ou en Arizona correspondent sensiblement aux taches sombres qu'on peut apercevoir sur la Lune et appelées, improprement, des mers). Qu'il fasse la dernière partie de son parcours à pied accentue l'impression que son identité s'ancre dans l'espace tellurique américain, renvoyant à toute une tradition américaine, à la fois littéraire et historique, qui procède d'une poétique de l'espace. Le roman se termine alors qu'il arrive à San Francisco. Les deux pieds dans le Pacifique, à l'extrémité ouest des États-Unis, il regarde l'horizon, "un vide qui s'étendait sans obstacle jusqu'aux rives de la Chine" (p. 364). Bouclant la boucle, Fogg renoue avec l'histoire du pays mais aussi avec son nom: "voir" la Chine veut dire renouer avec son prénom Marco (qui renvoie, comme le texte le dit explicitement, à Marco Polo), prénom enfoui depuis des années sous une simple initiale.

La mort de son oncle ("mon seul parent, mon unique relation à quelque chose de plus vaste que moi ", p. 13) déclenche chez Fogg un processus de transformations qui le propulse dans le tourbillon de l'Histoire: "L'histoire avait commencé sans moi, pour ainsi dire, et quand j'y entrai à mon tour, l'action était déjà engagée, elle échappait à tout contrôle" (p. 54). Dès lors, les mots n'ont plus de sens: perdre le contact avec sa famille équivaut ò perdre le contact avec le discours. Aux mots, Fogg substitue les formes qui l'entourent, tentant de les prendre à bras-lecorps pour ainsi dire, cherchant dans les plis du réel, dans les failles d'une réalité trop tangible pour être crédible, "quelque vérité fondamentale de l'univers" ( $p$. 49) à travers "le vaste univers sensoriel en contact avec [s]a peau. (p. 85). 
62

C'est à ce moment que Fogg se retrouve devant un jardin aux sentiers qui bifurquent, pour reprendre le titre de la nouvelle de Borges, voyant le monde se former et se déformer sous ses yeux, le chaos de son existence laissant entrevoir en palimpseste celui de la nation américaine. La catastrophe vécue par Fogg pendant toute la période où il erre dans Central Park ressemble à l'errance du gouvernement américain au Viet-Nam. C'est après avoir reçu sa convocation que la crise commence et elle se termine au moment où il se présente à l'examen médical. En réalité, à ce moment, il donne l'impression de revenir du Viet-Nam. La guerre, il l'a connue sur place, prenant le maquis dans Central Park, découvrant à son corps défendant une nouvelle culture chez ceuxlà même qui l'entouraient depuis toujours. Le choc de l'Asie, il en fera l'expérience plus tard, avec Kitty Wu.

Le rappel incessant de l'Histoire et de l'actualité américaine qui interfèrent dans sa propre vie se double de la présence de la littérature nationale. Donnons un exemple ponctuel parmi de nombreux cas de ce genre: Victor se transforme de clarinettiste en vendeur d'encyclopédie Humboldt, rappel du Humboldt's Gift de Saul Bellow, écrivain dont une bonne partie de la vie (notamment toute la jeunesse) s'est déroulée à Chicago où justement Fogg vivra avec son oncle.

Mais plutôt que d'énumérer les nombreuses références à la littérature américaine parsemant le roman, je m'arrêterai sur un cas particulièrement significatif pour diverses raisons. Il s'agit de Henry Adams, l'historien qui écrivit son autobiographie au début du $\mathrm{xx}^{\mathrm{e}}$ siècle, et du lien qu'on peut établir entre celui-ci et Fogg 20 .

L'importance de la filiation généalogique chez Adams s'impose, et pour cause : petit-fils et arrière-petit-fils de présidents des États-Unis, fils de diplomate (antiesclavagiste, son père est envoyé à Londres comme ministre plénipotentiaire pendant la guerre de Sécession), sa vie se confond avec l'histoire du pays. Alors que Henry Adams rompt avec sa famille (en rejetant certaines de ses valeurs), Fogg renoue malgré lui avec la sienne et

20 On connaît l'importance de la littérature américaine du xix ${ }^{\mathrm{e}}$ siècle dans l'œuvre de Paul Auster. Voir par exemple, de Malcolm Bradbury, The Modern American Novel, New York, Viking, 1992, p. 258-259. Voir aussi l'article de Daniel Canty dans les pages de ce numéro. 
(re)découvre le pays (et le paysage) américain à travers eux. Dans ce contexte, on ne s'étonnera pas que son père génétique, Julian Barber, comme Henry Adams, soit historien. Dans The Education of Henry Adams, sorte de roman d'apprentissage (cette autobiographie s'écrit à la troisième personne), Adams affirme que son éducation dans les meilleures écoles ne l'a pas préparé à affronter le monde, phrase que Fogg pourrait sans nul doute reprendre à son compte. Adams devient un peu le modèle américain dans lequel Fogg peut se mirer. C'est cependant la pensée qui traverse toute l'autobiographie d'Adams qui permet de le confronter à Moon Palace: "In The Education, embodying his theory of the acceleration of historical force, he describes the modern world as a "multiverse", leading to a metaphysics and science of multiple aspects and reactions." ${ }^{21}$ Dans son chapitre intitule "The Dynamo and the Virgin ", il tente de démontrer que l'électricité, source d'énergie socialement unificatrice, joue un rôle semblable à celui de la Vierge dans l'Europe médiévale. L'autobiographie "exhibit in Adam's own life the chaos of the Electric Phase, after the relative tranquillity of the Mechanical. Instead of the Virgin, man stands before the comfortless Dynamo" 22 .

Dans Moon Palace, Effing se souvient avec émotion de son passage à l'exposition Colomb en 1893 à cause de sa rencontre avec Tesla, grand promoteur de l'électricité (du courant alternatif plus précisément). Lors de sa première rencontre avec Fogg il lui dit: "En ce monde, tout est électricité, les objets animés comme les objets inanimés. Même les pensées produisent une charge électrique"(p. 131). L'électricité a imposé l'idée de relations entre les objets, d'un univers systémique, ouvert, où tout correspond.

Le chaos décrit par Adams renvoie à un univers scientifique moderne où l'entropie a fait son apparition. Le hasard dans Moon Palace, c'est le mouvement incessant d'un univers circulaire dans lequel le déterminisme n'est plus possible et où la leçon d'Adams a été comprise ${ }^{23}$. Pris de vertige, Fogg affirme: "Tout se mouvait

21 James D. Hart, American Literature, New York/Oxford, Oxford University Press, 1986, p. 118.

22 Marcus Cunliffe, The Literature of The United States, New York, Penguin Books, 1986, p. 274.

23 Cette "leçon" a été comprise par d'autres écrivains américains, comme le démontre Raymond Rouleau dans son analyse d'"Entropy". Voir "Entropy" de Thomas Pynchon: une dégradation féconde", à paraitre dans Les cabiers de recherche du CLADEST, printemps 1994. 
64

dans un flux constant, et si deux briques dans un mur pouvaient se ressembler très fort, à l'analyse elles ne se révéleraient jamais identiques. [...] Mon cerveau se prenait de palpitations lorsque je pensais à tout cela et me représentais les mouvements furieux et désordonnés des molécules, les incessantes explosions de la matière, les collisions, le chaos en ébullition sous la surface de toutes choses" (p. 151).

Par ailleurs, chez Adams, la référence à la Vierge concerne moins la religion qu'une source d'énergie dont elle serait le creuset et qui en ferait, culturellement, l'équivalent de l'électricité à l'aube du $\mathrm{xx}^{\mathrm{e}}$ siècle. En ce sens, de la science et de la technoscience à la religion, le pas serait moins grand qu'on pourrait le croire. Nous retrouvons là cette même tension fondamentale qui traverse tout le roman d'Auster où la conscience se voit déchirée entre des pulsions contraires, où matière et esprit sont toujours imbriqués:

J'essayais de me séparer de mon corps, de contourner mon dilemme en me persuadant qu'il n'existait pas. D'autres avant moi avaient suivi cette voie, et tous avaient découvert ce dont j'ai fini par m'apercevoir: l'esprit ne peut pas vaincre la matière, car sitôt qu'il se trouve sollicité exagérément, il se révèle lui aussi fait de matière (p. 45).

Cette tension entre raison et déraison, entre rationalité et irrationalité, ne prend pas sa source uniquement dans la pensée américaine. Encore une fois, un pont permet de traverser l'océan pour retourner aux sources de la pensée moderne.

Fogg revient toujours mal à l'aise de chez Chandler où il va vendre les livres de son oncle pour survivre. Le libraire ne s'intéresse qu'aux livres, à l'objet, et non pas à la valeur des mots. Pour Fogg, "les livres n'étaient pas tant le contenant des mots que les mots eux-mêmes, et la valeur d'un ouvrage donné dépendait de sa valeur spirituelle plutôt que de sa condition physique. Un Homère écorné, par exemple, avait plus de prix qu'un Virgile en parfait état; trois volumes de Descartes moins qu'un seul de Pascal. Ces distinctions étaient pour moi essentielles [...]" (p. 3738). Pascal, plus de trois fois plus important que Descartes? De la part de quelqu'un pour qui les mots ont du poids, l'affirmation est décisive. En arrivant chez Effing où il s'installe, déballant ses affaires, il précise ne posséder qu'un livre, une édition de poche des Pensées de Pascal. Par contre, lorsque Effing lui dicte ses souvenirs, il souligne être parti pour la France, en septembre 1920, 
sur le s.s. Descartes. Façon implicite de dire que Descartes n'a qu'à retourner d'où il est venu...

À l'ars inveniendi de Descartes, Pascal oppose un ars demonstrandi, méthode synthétique qui, à l'ère de la postmodernité, dans le monde quantique et cybernétique où nous vivons, paraît plus défendable. Pour Anthony Wilden par exemple,

la "révolution" cartésienne commit l'erreur cruciale (pour nous), erreur absolutiste et analytique, d'accorder gratuitement un statut ontologique privilégié aux entités ("substances") plutôt qu'aux relations ("attributs", "accidents"). [...] Pascal, ce penseur paradoxal qui s'opposa si violemment à Descartes, ne le savait que trop bien: l'erreur épistémologique consistait à enfermer la réalité du "à la fois-et" (système ouvert) dans une logique du "ou bien/ou bien" (système clos) ${ }^{24}$.

Dans le tourbillon de l'Histoire, dans le tourbillon du temps, l'individu apparaît fragile, incapable de se situer ou de se définir, point minuscule par rapport à l'infini du cosmos. En paraphrasant ainsi Pascal, on voit se dessiner les contours de Fogg, cherchant les lois secrètes, les liens, les réseaux d'association que les lois de la logique et de la raison cartésiennes rendent invisibles. C'est justement en s'abandonnant à une quête insensée que Fogg parvient à retrouver les traces de son passé. Ce qui s'impose comme une sêrie de hasards résulte au fond d'une autre forme de logique, transversale, un renoncement aux systèmes clos d'énergie où les choses s'ajoutent les unes aux autres mais de manière statique. L'idéalisme d'un monde stable et saisissable n'a plus cours. Dans la deuxième maxime du Discours de la métbode, Descartes affirme qu'il faut "être le plus ferme et le plus résolu en [ses] actions" et, pour mieux rendre compte de cette idée, il utilise la métaphore des voyageurs qui, "se trouvant égarés en quelque forêt, ne doivent pas errer en tournoyant tantôt d'un côté, tantôt d'un autre, ni encore moins s'arrêter en une place, mais marcher toujours le plus droit qu'ils peuvent vers un même côté "25. On ne pourrait imaginer plus éloigné de ce que vit Fogg...

Peut-on alors parler d'un rejet complet de la pensée cartésienne? Face à l'inextricable incohérence du monde, Fogg ne

24 Anthony Wilden, Système et structure, Montréal, Boréal express, 1983, p. 216217.

25 Descartes, Discours de la méthode, Paris, Gallimard, "Le Livre de poche", 1970 , p. 52. 
66

semble pourtant pas partager la confiance de Pascal en la grâce arbitraire du hasard. Recommencer à zéro pour lui, ce qu'il affirme à plusieurs reprises, signifie faire face au réel, au concret, au prosaïsme. Mais, pris dans la spirale du mouvement qu'il a luimême amorcé, les événements dérapent souvent. Pourtant, régulièrement, il tente de ramener les choses du côté de la raison. Car sans le fil de sa raison, sans sa volonté parfois obsessive de rationnaliser (dans des situations où cela apparaît d'ailleurs souvent paradoxal), il n'y aurait plus de contrepoids à cet univers sans balises, où tout ne semble tenir qu'aux relations entre les choses et où les valeurs deviennent obsolètes. La mémoire a besoin de classer et de faire des choix, de hiérarchiser et de tenir compte de priorités. Sinon il ne reste que l'oubli. Le vaste mouvement brownien dans lequel tournoie l'univers est par définition un mouvement totalement dépourvu de mémoire.
À la confusion des chemins on pourrait plus justement compa- rer la confusion très particulière engendrée par le phénomène de l'oubli. De même que l'insignifiance se définit non par le manque mais par la prolifération des chemins, de même l'oubli se caractérise non pas par une perte du souvenir mais bien par une omniprésence des souvenirs, par la masse indistincte de tous les souvenirs [...] Dans l'état d'oubli je ne trie plus et ai alors face à moi, sur un pied d'égalité, tous mes souvenirs. Comment choisir? Comment s'orienter dans cette masse qui n'est même pas un dédale, comme le labyrinthe? Il n'y a plus de chemins, pas même de fausses directions. Il n'y a plus de di- rections, plus de poteaux indicateurs. Ou plutôt, il y a bien encore des signaux mais ceux-ci sont devenus dérisoires. ${ }^{26}$

C'est parce que la raison chancelle qu'il faut s'y tenir. Ce qui signifie s'accrocher à la mémoire, à l'Histoire, à une narration qui peut donner le sens d'une continuité dans un monde prisonnier du discontinu et de l'aléatoire.

Plus que la géographie et l'Histoire, ce sont des mythes, propres aux États-Unis, qui abondent dans Moon Palace: de la conquête de l'Ouest au baseball, de la vie de différentes tribus indiennes jusqu'à la notion de progrès. Par contre, l'image de la jeunesse et de la nouveauté, qu'on associe spontanément aux Américains (c'est un cliché commode), prend, si on peut dire, un coup de vieux. Jamais le personnage de Fogg n'apparaît juvénile.

26 Clément Rosset, Le réel. Tratté de l'idiotie, Paris, Minuit, 1977, p. 18. 
Plutôt qu'un personnage psychologisé, il surgit comme le mobile, la raison d'être d'un roman qui vise d'abord à indiquer des filiations, de la modernité occidentale à l'histoire (culturelle et politique) américaine. Détonateur d'un univers extrêmement cohérent et sophistiqué, il permet d'en suivre tous les méandres et d'y voir clair, de s'y retrouver parmi les sinuosités et les lacets enchevêtrés d'un texte donnant pourtant l'impression d'être relativement linéaire.

"Comme me l'avait un jour lointain expliqué oncle Victor, se souvient Fogg, une conversation ressemble à un échange de balles. Un bon partenaire vous envoie la balle droit dans le gant, de sorte qu'il vous est presque impossible de la rater; quand c'est à lui de recevoir, il rattrappe tout ce qui arrive de son côté, même les coups les plus erratiques et les plus incompétents" (p. 118). Cet éloge de la conversation vue comme un "échange sportif " rappelle le roman lui-même. Prolifération de discours sans certitude, sans "vérité", Moon Palace rappelle la parole kaïrotique des sophistes où le sens surgit peu à peu de la circulation des arguments entre locuteurs. La narration est une dérive qui ne propose pas de direction privilégiée mais s'ouvre à un espace discursif fait de paradoxes et de tensions où le monde contemporain se mire dans son passé. À travers Fogg se développe une conscience du monde et de l'Histoire. C'est ainsi que Moon Palace fait du savoir et de la connaissance les clés de la compréhension de tout espace matériel auquel les personnages du roman se trouvent confrontés. 